\title{
Engineering of a bacterial tyrosinase for improved catalytic efficiency towards D- tyrosine using random and site directed mutagenesis approaches ${ }^{\dagger}$
}

\section{Susan Molloy ${ }^{1}$, Jasmina Nikodinovic-Runic ${ }^{1 \#}$, Leona B. Martin ${ }^{1}$, Hermann Hartmann², Francisco Solano $^{3}$, Heinz Decker ${ }^{2}$ and Kevin E. O'Connor ${ }^{1 *} \mathrm{~S}$}

${ }^{1}$ School of Biomolecular and Biomedical Sciences, Ardmore House, University College Dublin, Belfield, Dublin 4, Ireland. ${ }^{2}$ Institute for Molecular Biophysics, University of Mainz, Johannes Gutenberg-Universität D 55099 Mainz, Germany. ${ }^{3}$ Department of Biochemistry and Molecular Biology B, University of Murcia, 30100 Murcia, Spain

${ }^{\#}$ Present address: Institute of Molecular Genetics and Genetic Engineering, University of Belgrade, Vojvode Stepe 444a, P.O.Box 23, 11000 Belgrade, Serbia

S This article contains Online Supplementary Material

* Correspondence to: Kevin O'Connor

Tel.: + 353 (1) 716-1307; FAX: +353 (1) 716-1183; E-mail: kevin.oconnor@ucd.ie

Running title: Engineering bacterial tyrosinase towards D-tyrosine

\footnotetext{
${ }^{\dagger}$ This article has been accepted for publication and undergone full peer review but has not been through the copyediting, typesetting, pagination and proofreading process, which may lead to differences between this version and the Version of Record. Please cite this article as doi: [10.1002/bit.24859]
}

Additional Supporting Information may be found in the online version of this article.

(C) 2013 Wiley Periodicals, Inc.

Received November 13, 2012; Revision Received January 17, 2013; Accepted January 22, 2013 


\section{Abstract}

The tyrosinase gene from Ralstonia solanacearum (GenBank NP518458) was subjected to random mutagenesis resulting in tyrosinase variants (RVC10 and RV145) with up to 3.2 fold improvement in $\mathrm{k}_{\text {cat}}, \quad 5.2$ fold lower $\mathrm{Km}$ and 16 fold improvement in catalytic efficiency for D-tyrosine. Based on RVC10 and RV145 mutated sequences, single mutation variants were generated with all variants showing increased $\mathrm{k}_{\mathrm{cat}}$ for D-tyrosine compared to the wild type (WT). All single mutation variants based on RV145 had a higher kcat and Km value compared to the RV145 and thus the combination of 4 mutations in RV145 was antagonistic for turnover, but synergistic for affinity of the enzyme for D-tyrosine. Single mutation variant 145 _V153A exhibited the highest (6.9 fold) improvement in kcat and a 2.4 fold increase in Km compared to the WT. Two single mutation variants, C10_N322S and C10_T183I reduced the Km up to 2.6 fold for D-tyrosine but one variant 145_V153A increased the Km 2.4 fold compared to the WT. Homology based modelling of $R$. solanacearum tyrosinase showed that mutation V153A disrupts the van der Waals interactions with an $\alpha$-helix providing one of the conserved histidine residues of the active site. The kcat and $\mathrm{Km}$ values for $\mathrm{L}-$ tyrosine decreased for RV145 and RVC10 compared to the WT. RV145 exhibited a 2.1 fold high catalytic efficiency compared to the WT which is a 7.6 fold lower improvement compared to Dtyrosine. RV145 exhibited a 3 fold higher monophenolase:diphenolase activity ratio for D-tyrosine:D-

$\begin{array}{llllll}\text { DOPA } & \text { and } & \text { a } & 1.4 & \text { fold } & \text { higher }\end{array}$

L-tyrosine:L-DOPA activity ratio compared to the WT.

Key words: Tyrosinase, D-tyrosine, random mutagenesis, site specific mutagenesis, Enzyme catalysis, Homology modeling 


\section{Introduction}

Tyrosinases (E.C.1.14.18.1) are copper containing oxidase enzymes and along with hemocyanins and catechol oxidases, are classified as type 3 copper proteins (Decker et al. 2006; Decker and Tuczek 2000; van Holde et al. 2001). Tyrosinases possess two copper atoms at their active site (CuA and $\mathrm{CuB}$ ). These $\mathrm{Cu}$ atoms are each coordinated by three conserved histidine residues located in a 'four $\alpha$ helix bundle' (Matoba et al. 2006; Rolff et al. 2011; Solomon et al. 1996). In the presence of molecular oxygen, tyrosinases display both monophenol and o-diphenol oxidase activity converting phenols to catechols and subsequently converting catechols to quinones (Fig. 1) (Claus and Decker 2006; Halaouli et al. 2006; Sánchez-Ferrer et al. 1995; Solomon et

al. 1996). The quinones nonenzymatically polymerise to yield red, brown or black pigments (Espin et al. 1997; Garcia-Molina Mdel et al. 2012).

There has been widespread interest in tyrosinases as potential biocatalysts as they are readily available, have a broad substrate range and do not require the addition of exogenous cofactors (Burton 2003; Espin et al. 2001; Jus et al. 2012). The focus of the vast majority of kinetic and biocatalysis studies with tyrosinases has been with L-tyrosine, with limited reports on the D-enantiomer (Espin et al. 1998a; Wilcox et al. 1985) and a complete absence of enzyme engineering towards D-tyrosine. Ltyrosine is a key target for biological oxidation as L-DOPA is used in the treatment of Parkinson's disease, a degenerative disorder resulting from a loss of dopamine production in the brain, leading to involuntary movements or tremors (Lipski et al. 2011). D-DOPA, the product of tyrosinase oxidation of D-tyrosine, has been suggested as the better alternative to L-DOPA in the treatment of Parkinson's disease (Kawazoe et al. 2007; Moses et al. 1996). While fungal tyrosinases have been reported to have high monophenolase activity with D-tyrosine, the diphenolase activity is over 10 fold higher than the monophenolase activity, resulting in the through conversion of D-DOPA to dopaquinone making these tyrosinases undesireable for the catechol production (Espin et al. 1998b; Wilcox et al. 1985). A gene 
encoding tyrosinase (GenBank Accession number NP518458) was identified in the genome sequence of $R$. solanacearum as putatively coding for a polyphenol oxidase (Hernandez-Romero et al. 2005). It was subsequently recombinantly expressed and displayed tyrosinase like activity (Hernandez-Romero et al. 2006). Unlike all other tyrosinases, the enzyme from $R$. solanacearum is the only known tyrosinase with a monophenolase activity greater than its diphenolase activity and thus it has great potential as a biocatalyst for the production of D-DOPA (Hernandez-Romero et al. 2006; Salanoubat et al. 2002). However, we have found that the enzyme exhibits a low rate of activity and low affinity with D-tyrosine. The current study reports on improvements to the activity, affinity, and catalytic efficiency of tyrosinase from R. solanacearum towards D-tyrosine by random and site directed mutagenesis, while maintaining or improving its monophenolase to diphenolase activity ratio and also identifying the key amino acids responsible for the improved activity and affinity.

\section{Materials and Methods}

\section{General materials and Methods}

All chemicals, substrates and media components were purchased from Sigma-Aldrich (Dublin, Ireland) or Fisher Scientific (Dublin, Ireland) unless otherwise stated, and used without further purification. Strains, plasmids and primers used in this study are listed in Supplemental Table I. For culture propagation, Luria-Bertani (LB) or M9 minimal medium were used (Sambrook et al. 1989). M9 medium was supplemented with casamino acids $(0.5 \%, \mathrm{w} / \mathrm{v})$. Carbenicillin $\left(50 \mu \mathrm{g} \mathrm{ml}^{-1}\right)$ was routinely used to select for ampicillin resistance $\left(a m p^{R}\right)$. Stock cultures of all strains used in this study were maintained at $-80^{\circ} \mathrm{C}$ in LB broth with glycerol $(20 \%, \mathrm{v} / \mathrm{v})$. 


\section{DNA Techniques and Plasmid Construction}

Standard recombinant DNA techniques were performed as previously described (Sambrook et al. 1989). Tyrosinase was amplified by PCR using chromosomal DNA from $R$. solanacearum as template and DNA Engine Thermal Cycler (Bio-Rad, Hercules, USA). The $1.5 \mathrm{kbp}$ fragment generated by PCR was digested with XhoI and EcoRI (Invitrogen, Paisley, UK), and ligated into pRSETb using T4 DNA ligase (Stratagene, La Jolla, USA) to generate pRSET-tyrR0 (Supplemental Table I). To verify recombinant plasmid constructs DNA sequencing was conducted by GATC Biotech (Konstanz, Germany), using appropriate primers (Supplemental Table S1). Sequence data were aligned and compared to GenBank database using BLAST programme (Altschul et al. 1997). Sequences of variants obtained and described were deposited in GenBank under Accession numbers JX272609JX272630.

\section{Random and Site-directed Mutagenesis}

In vitro random mutagenesis of tyrosinase was performed using a GeneMorph ${ }^{\circledR}$ II EZClone Domain Mutagenesis kit (Stratagene, La Jolla, CA) according to the manufacturer's instructions. Error prone PCR (epPCR) using $30 \mathrm{ng}$ of plasmid template (high mutation frequency) was carried out to generate a megaprimer, which was used to amplify the rest of the plasmid generating the library of recombinant plasmids with mutated tyrosinase fragment. The resulting plasmids were used to transform E. coli BL21(DE3) cells, which were then plated onto large LB agar plates (150 x $20 \mathrm{~mm}$ Petri dish size) containing carbenicillin $\left(50 \mu \mathrm{g} \mathrm{ml}^{-1}\right)$ and incubated for $18 \mathrm{~h}$ at $37^{\circ} \mathrm{C}$. Site directed mutagenesis (SDM) was carried out using a QuikChange ${ }^{\circledR}$ Site-Directed Mutagenesis Kit (Stratagene, La Jolla, CA) according to the manufacturer's instructions using appropriate primers (Supplemental Table I) for the introduction of single mutations. The new plasmids (Supplemental Table I) were also transformed into E. coli BL21(DE3). DNA sequencing was verified by GATC Biotech (Konstanz, Germany). 


\section{Screening Whole Cells Expressing Tyrosinase Variant Genes.}

Libraries of E. coli BL21 (DE3) cells were screened both on agar plates and in liquid media (Supplemental Fig. S1). M9 agar plate supplemented with $1 \mathrm{mM}$ D-tyrosine and $50 \mu \mathrm{g} \mathrm{ml}{ }^{-1}$ carbenicillin were incubated at $37^{\circ} \mathrm{C}$ for up to $24 \mathrm{~h}$ and at room temperature for another $24 \mathrm{~h}$. During this period colonies were regularly visually examined for improved melanin production rate and intensity in comparison to E. coli expressing wild type tyrosinase. Cells grown in liquid medium overnight at $37^{\circ} \mathrm{C}$ were centrifuged and resuspended in $180 \mu \mathrm{l}$ of $50 \mathrm{mM}$ potassium phosphate buffer containing $1 \mathrm{mM}$ D-tyrosine. $\mathrm{OD}_{600}$ of each well was measured before incubating for 1 to $4 \mathrm{~h}$ at $37^{\circ} \mathrm{C}$ with shaking at $200 \mathrm{rpm}$. The assay plate was then centrifuged at $3200 \times g$ in a benchtop centrifuge (Eppendorf $5804 \mathrm{R}$, Hamburg, Germany) at $4^{\circ} \mathrm{C}$ for $15 \mathrm{~min}$, and the supernatant removed. $200 \mu 1$ of the supernatant was transferred to a new 96-well plate and the absorbance at $400 \mathrm{~nm}$ was determined for all wells. Cultures showing increased pigment formation rates greater than the positive control, in liquid assays $\left(\mathrm{OD}_{400} / \mathrm{OD}_{600}\right)$ and on agar plates (visual screening), were selected for determination of enzyme activity. Recombinants with the highest rates of melanin formation were cultured for plasmid isolation and DNA sequencing (GATC Biotech, Konstanz, Germany).

\section{Recombinant Tyrosinase Purification}

All recombinant strains (Supplemental Table I) were grown in $400 \mathrm{ml} \mathrm{LB}$ broth at $37^{\circ} \mathrm{C}$ with shaking at $200 \mathrm{rpm}$ for $4 \mathrm{~h}$ or until $\mathrm{OD}_{600}$ of 0.4 was reached. Cultures were cooled on ice for 20 min before inducing with $0.5 \mathrm{mM}$ (final concentration) Isopropyl- $\beta$-D-thiogalactopyranoside (IPTG). Following induction, cultures were incubated at $30^{\circ} \mathrm{C}$ shaking at $200 \mathrm{rpm}$ for up to $18 \mathrm{~h}$. The cells were harvested by centrifugation at $3600 \times g$ for $12 \min$ (Eppendorf $5804 \mathrm{R}$ benchtop centrifuge). The cell pellet was then stored at $-20^{\circ} \mathrm{C}$ awaiting protein purification. Cells were lysed and the desired tyrosinase was purified as described previously (Hume et al. 2009). Fractions ( $2 \mathrm{ml})$ were collected and analyzed by SDS-PAGE (Laemmli 1970) under denaturing conditions with $10 \%$ and $4 \%$ acrylamide (w/v) in 
resolving and stacking gels, respectively. Protein concentration was determined using the bicinchoninic acid method (Smith et al. 1985).

\section{Enzyme Kinetic Assays}

Kinetic studies on the oxidation of D-tyrosine and L-tyrosine were performed as described previously (Martin et al. 2008). The assay medium $(0.2 \mathrm{ml})$ contained $50 \mathrm{mM}$ phosphate buffer, $\mathrm{pH} 7.0$, substrate and $0.002 \mathrm{mg} /$ assay of protein, which was added last to start the reaction. Substrate concentration was varied between 0.03125 and $20 \mathrm{mM}$. $\mathrm{k}_{\text {cat }}$ was determined using the $\mathrm{V}_{\max }$ data. Initial rates were obtained by fitting linearly the change in absorbance $\left(\Delta \mathrm{A}_{400 \mathrm{~nm}}\right)$ versus time plots. Kinetic parameters were obtained as described previously (Martin et al. 2008).

\section{Monophenolase:diphenolase Activity Assay}

A $5 \mathrm{ml}$ assay contained, $50 \mathrm{mM}$ potassium phosphate buffer $(\mathrm{pH} 7.0), 5 \mathrm{mM}$ substrate and $0.01 \mathrm{mg} \mathrm{ml}^{-1}$ purified protein (final concentration) of WT and selected variants. Protein was added last to start the reaction. Reference assays were carried out in the absence of either protein or substrate, and replaced with $50 \mathrm{mM}$ potassium phosphate buffer ( $\mathrm{pH}$ 7.0). The assays were incubated shaking at $30^{\circ} \mathrm{C}$ at 200 rpm for $60 \mathrm{~min}$. $450 \mu \mathrm{l}$ samples were withdrawn at various time points and immediately added to $50 \mu 1$ of ice-cold $1 \mathrm{M}$ hydrochloric acid and incubated on ice awaiting centrifugation. Samples were centrifuged $(12000 \times \mathrm{g})$, filtered $(0.2 \mu \mathrm{m}, 4 \mathrm{~mm}$, nylon syringe filter; Sarstedt, Germany) and substrate depletion/product formation analysed by HPLC.

\section{High Performance Liquid Chromatography (HPLC)}

The HPLC apparatus included a C-18 Hyperclone ODS $5 \mu$ column (250 mm x $4.6 \mathrm{~mm}$ ) (Phenomenex, Ireland) interfaced with a Hewlett Packard HP 1100 series instrument equipped with an Agilent 1100 series diode array detector. Metabolites were eluted under isocratic conditions using a mixture of phosphoric acid $(0.1 \%, \mathrm{v} / \mathrm{v})$ and methanol as the mobile phase. The ratio of methanol to phosphoric acid was 15:85 for L-tyrosine and D-tyrosine. The flow rate was $0.8 \mathrm{ml} \mathrm{min}^{-1}$ and the injection volume 
was $20 \quad \mu$ l. $\quad$ The retention time $\quad$ was $7.9 \quad \min$ for

L-tyrosine and D-tyrosine and 4.9 min for L-DOPA and D-DOPA.

\section{Model Building}

BLASTP (Altschul et al. 1997) was used to search the Protein Data Bank (PDB) for homologous proteins with known 3D structures. Significant hits were only found for the N-terminal part of the $R$. solanacearum tyrosinase from MET1 to LYS319. The proteins with the best scores are: 1) polyphenol oxidase from the grape Vitis vinifera (PDP ID: 2P3X) with an E-Value of $3 \cdot 10^{-23}$ and a sequence identity (SI) of $27 \%$; ) catechol oxidase from the sweet potatoes Ipomoea batatas (PDB ID: 1BT1, chain A with E-Value of $2 \cdot 10^{-20}$ and SI of $25 \%$ ).

For the C-terminal fragment, spanning residues 320 to 496 , no homologous protein with known 3D structure could be detected with the BLAST algorithm. The fold recognition server PHYRE (Bennett-Lovsey et al. 2008); www.sbg.bio.ic.ac.uk/phyre) found as weakly related folds the head domain of phage bIL170 (PDB ID: 2FSD) and the C-terminal domain of the Octopus doflenii hemocyanin, but with rather high E-values of 14 and 22 respectively. Both were beta barrel domains. Additionally, none of these two folds had any similarity with the sequence of the $R$. solanacearum tyrosinase in the amino acid range from 320 to about 380, where some of the mutations are located. Therefore we generated 3D models only for the N-terminal part from MET1 to LYS319.

Because it is well known that inaccuracies in homology models are mostly due to alignment errors (Melo and Šali 2007), we have produced different alignments using different locally installed programs and WEB-server: ClustalX (Larkin et al. 2007), T-coffee (Notredame et al. 2000), align2d from the MODELLER package (Šali and Blundell 1993). In addition, we tried different parameters and generated pair wise and multiple alignments. The sequence of $R$. solanacearum tyrosinase was aligned pair wise to the best scoring templates from the BLAST search 2P3X, 1BT1 and multiple aligned to $2 \mathrm{P} 3 \mathrm{X} / 1 \mathrm{BT} 1$. While the core region around the six essential histidines, which binds the two 
copper ions, aligned perfectly the low sequence identity of about $25 \%$ for the best scoring template resulted in poor alignment of other regions.

For the homology modeling we used the program MODELLER 9. This program creates models for the target sequence by satisfaction of spatial restraints derived from the coordinates templates, which were homologous to the target. The generated 3D models for the target sequence were subsequently annealed by molecular dynamic simulations. Models could be produced based on single templates or alternatively on multiple templates. In the production runs we generated models with the single templates $2 \mathrm{P} 3 \mathrm{X}$ and $1 \mathrm{BT} 1$ and with the combination of these two structures. For each of the different alignments, we have generated about 50-200 different models.

Assessment of model quality was performed with the score values of the MODELLER program, the GA341 score and the normalized DOPE score (Melo and Šali 2007) and the models were ranked according to these scores. While the GA341 score includes the sequence identity between template and target and is therefore meaningful only for homology models, the normalized DOPE score can also be given for the experimentally determined structures of the templates. Unfortunately, the best scores are very similar for rather different models. The three models shown in Fig. 3A, all have a GA341 score between 0.97 and 0.99 , near the optimal value of 1.0. In addition, all the three models have a normalized DOPE score of about -1.1. This value can be compared with the worst values of all produced models which are about +0.3 and with the corresponding DOPE scores for the X-ray structures $2 \mathrm{P} 3 \mathrm{X}$ and 1BT1, which are -1.96 and -2.17 , respectively. Model 1 is based on an alignment with Clustal, model 2 on the alignment with align $2 \mathrm{~d}$ and model 3 is the result of an alignment with Tcoffee. For all three alignments in Fig. 3A we used the default parameters of the alignment programs and the 3D models were generated with the multiple template mode of MODELLER using the templates 2P3X and 1BT1. The rms difference for all $\mathrm{C} \alpha$ atoms of the three models is $2.9 \AA ̊$ and $1.4 \AA$ omitting 31 residues in loop regions not containing mutation sites. 


\section{Results}

\section{Random Mutagenesis of Tyrosinase from $R$. solanacearum with D-tyrosine as the Target}

\section{Substrate}

Over 10000 E. coli colonies expressing tyrosinase variants generated by random mutagenesis were screened for pigment formation on solid and in liquid media in the presence of D-tyrosine (Supplemental Fig. S1). As we observed a large variation in protein expression in the presence of IPTG we minimised that variation by screening colonies in the absence of the inducer IPTG (allowing only leaky but consistent expression). The best variants were selected for purification and kinetic analysis based on the rate and intensity of colour formation on agar plates in conjunction with the quantitative analysis of melanin formation in liquid assays. Comparison of relative mobility of the WT tyrosinase and variant proteins with that of protein standards indicated that a molecular mass was approximately $55 \mathrm{KDa}$ by SDS-PAGE (Supplemental Fig. S2). The catalytic properties of the purified WT enzyme and variants of tyrosinase were investigated with D-tyrosine as a substrate (Table I). Random mutation variants, RVC10 and RV145, exhibited a 2.1 and 3.2 fold increase in $\mathrm{k}_{\text {cat }}$, respectively, compared to the WT while the $K_{m}$ was 3.2 fold and 5.2 fold lower, respectively, than the WT. The overall catalytic efficiency for the variants RVC10 and RV145 had improved 6.7 and 16 fold compared to the WT for D-tyrosine.

Sequence analysis showed that four different amino acids were altered in both variants RVC10 and RV145 (Fig. 2, Supplemental Fig. S3). In the RVC10 variant, two conservative amino acid changes occurred at N322S (polar to polar) and T359M (amphipathic to amphipathic) while the other two mutations (T183I (amphipathic to hydrophobic) and F185Y (hydrophobic to amphipathic)) were relatively non-conservative amino acid changes (Supplemental Fig. S4). In random variant RV145 a hydrophobic phenylalanine was substituted with a more hydrophilic tyrosine (Fig. 2, Supplemental Fig. S3). The amino acid changes V153A and L330V were conservative while D317Y substitution was non 
conservative with a hydrophilic linear side chain replaced with an amphipathic aromatic side chain (Bugg 1997; Petsko and Ringe 2004). The majority (five) of amino acid changes occurred within the N-terminus while only three amino acid changes (N322S, L330V and T359M) occurred within the Cterminus. The first 50 amino acids in the N-terminus appear to encode a leader sequence but none of the mutations occurred in this sequence.

\section{Introduction of Single Amino Acid Substitutions into Tyrosinase of $R$. solanacearum Based on Random Variant RVC10 and RV145 with D-tyrosine as the Target Substrate}

In order to investigate the effects of each amino acid change on enzyme activity with D-tyrosine as a substrate, single mutations were introduced into the WT DNA of tyrosinase by SDM, to generate variants with single amino acid changes (Fig. 2A). The $\mathrm{k}_{\mathrm{cat}}$ for each single mutation variant was higher than both random variant RVC10 and the WT with D-tyrosine (Table II). While C10_T183I and C10_N322S had 1.8 fold and 2.6 fold lower $K_{m}$ values, respectively, than the WT both were higher than random variant RVC10. C10_F185Y and C10_T359M had similar $K_{m}$ values to the WT (Table II). The overall catalytic efficiency for all single mutation variants was higher than the WT. The catalytic efficiency for C10_T183I and C10_N322S variants was increased from both the WT and random variant RVC10. Variant C10_N322S displayed the highest catalytic efficiency $\left(30 \mathrm{~min}^{-1} \mathrm{mM}^{-1}\right)$ with a 1.5 fold and a 10 fold increase over random variant RVC10 and the WT respectively (Table II).

Single mutations were introduced into the WT DNA of tyrosinase from $R$. solanacearum by SDM to generate variants baed on RV145 (Fig. 2B). These single mutation variants had a higher turnover number than the WT and RV145 (Table II). 145_V153A had the highest turnover number which was 6.8 and 2.1 fold higher than the WT and random variant RV145, respectively. Although 145_Y119F displayed the lowest turnover number of single mutations variants of random variant 145 , it was still 4 fold higher than the WT. The $K_{m}$ values of all single mutation variants were higher than random variant RV145 (1.2 mM) (Table II). 145_Y119F was the only single mutation variation of random mutant 145 with a $K_{m}(5.1 \mathrm{mM})$ lower than the WT $(6.2 \mathrm{mM})$. The catalytic efficiency of each 
single mutation variant was higher than the WT but significantly reduced from random variant RV145 (Table II).

Kinetic Analysis of WT and Selected Tyrosinase Variants with L-tyrosine as the Target Substrate

Within the range of error, the turnover number was similar for the WT, RVC10 and C10_N322S variants with L-tyrosine. Random variant RV145 (54.5 $\left.\mathrm{min}^{-1}\right)$ had a turnover number 1.5 fold lower than the WT $\left(84.8 \mathrm{~min}^{-1}\right)$. However, the $K_{m}$ values for all variants were lower than the WT $(2.1 \mathrm{mM})$ (Table III). All variants displayed a higher catalytic efficiency for L-tyrosine compared to the WT. The highest catalytic efficiency was displayed by random variant RV145 (85.2 $\left.\mathrm{min}^{-1} \mathrm{mM}^{-1}\right)$, which was 2.2 fold higher than the WT $\left(40.4 \mathrm{~min}^{-1} \mathrm{mM}^{-1}\right)$. While the amino acid changes result in improvements in activity and catalytic efficiency the magnitude of the improvements is much lower for L-tyrosine compared to D-tyrosine where the maximum increase in catalytic efficiency displayed by random variant RV145 was 16 fold higher than the WT (Table I and Table IV).

Determination of the Monophenolase:diphenolase Activity Ratio of the WT and Selected Tyrosinase Variants

Tyrosinase from $R$. solanacearum has a uniquely high MDR (Hernandez-Romero et al. 2006). The MDR of the WT and selected tyrosinase variants (RVC10, RV145, and C10_N322S) was measured to investigate if the random variants had maintained this ratio (Table IV). HPLC analysis showed that the random variants had an improved MDR for both D- and L-enantiomers of tyrosine and DOPA (Table IV). The MDR values of the two random variants, RVC10 and RV145, for the D-enantiomer of tyrosine and DOPA were improved 2 fold and 3 fold respectively (Table IV) while the MDR values for the L-enantiomer of tyrosine and DOPA were between 1.3 and 1.5 fold higher for variants compared to the WT (Table IV). 


\section{Discussion}

The screening of E. coli libraries expressing variants of the tyrosinase gene was achieved using colorimetry where the appearance of the melanin product in the shortest time period was taken as a key indicator of improved tyrosinase activity. There is an inherent risk in such a strategy that the tyrosinase variants with improved diphenolase rather than improved monoophenolase activity could be selected. However the rapid appearance of a dark colour cannot be achieved without a rapid production of catechol due to monophenolase activity. Thus we reasoned that while the risk of selecting a variant with improved diphenolase activity was possible one was as likely to select a variant with improved monophenolase activity. Such a strategy was justified as the random mutants selected using the colorimetric screen had greater improvements in monophenolase compared to diphenolase activity.

Tyrosinase (GenBank Accession number NP_518458) from R. solanacearum was evolved by random and site directed mutagenesis towards D-tyrosine producing variants with up to 3.2 fold improvement in turnover number, 6.2 fold increase in affinity and 16 fold improvement in catalytic efficiency compared to the WT. The amino acids identified in the improved variants have not previously been identified as amino acids influencing tyrosinase activity. This is the first time, to our knowledge, that a tyrosinase enzyme has been engineered to show improved activity towards Dtyrosine and that the amino acid changes result in greater improvement in enzyme activity with the Denantiomer compared to the L-enantiomer.

Single mutation variants C10_T183I and C10_N322S would appear to be responsible for the decrease in $K_{m}$ observed for the random RVC10 variant as the other two single mutation variants C10_F185Y and C10_T359M, did not have any effect on the $K_{m}$ compared to the WT (Table II). Single mutation variants of random variant RV145 exhibited higher $K_{m}$ values compared to RV145 (Table II) suggesting that the four mutations combined in RV145 have a synergistic effect on the affinity of the enzyme for D-tyrosine. It has been shown that neutral and deleterious mutations can 
accumulate in positive variants during directed evolution (random mutagenesis) to form epistatic relationships with beneficial mutations (Salverda et al. 2011). Beneficial mutations can often rely on the presence of more stabilising, but neutral or deleterious mutations (Bloom and Arnold 2009). Furthermore all single mutation variants based on RV145 had a higher turnover number than the WT and RV145. It is possible that the four mutations combined in RV145 have antagonistic effects on the turnover number compared to the single mutation variants (Mildvan et al. 1992).

The single mutation at position $153\left(145_{\text {V V } 153 A)}\right.$ ) resulted in a 6.8 fold increase in $\mathrm{k}_{\mathrm{cat}}$ and a 2.4 fold increase in $K_{m}$ compared to the WT (Table II). The amino acid change from valine to alanine results in the substitution of a hydrophobic branched side chain with a shorter and less hydrophobic side chain. For this mutation the functional changes can be modelled since a reliable 3D model is available for this part of the tyrosinase (P51 to K319). A modelling strategy was applied based on three different alignments which resulted in a very good superposition of the catalytic centre (Fig. 3A). Amino acid site 153 is located on a loop which is close to the N-terminal part of an $\alpha$-helix containing the conserved residue His 253 of the active site which complexes $\mathrm{CuB}$ (Fig. 3). The distance from V153 to P247, the nearest amino acid of the $\alpha$-helix, is $3.6 \AA$ (Fig. 3B) whereas the distance from mutated A153 to P247 increases to $4.9 \AA$ (Fig. 3C). By creating an extra space in the presence of A153, it is possible that the hydrophobic van der Waal interactions (Betts and Russell 2003) have been disrupted, providing less steric hindrance with the shorter side chain of alanine and giving the $\alpha$-helix more freedom and flexibility to move. $\alpha$-helices are rigid structures (Bugg 1997; Petsko and Ringe 2004) which will move entirely if one amino acid is forced to adjust in some way. Thus, H253 may move within the active site as a result of the $\alpha$-helix movement, which may induce changes in the distances between the two copper atoms. This may influence the orientation of the neighbouring $\mathrm{H} 231$ which seems to be responsible for the correct orientation of the substrate through a $\pi$ - $\pi$-interaction as proposed previously (Decker et al. 2006). The kinetic analysis of 145_V153A, showed a higher 
turnover number compared to the WT (126- $\mathrm{min}^{-1}$, Table II) but a decreased affinity. Previous work involving SDM of enzymes have shown that distal mutations can have an effect on activity and substrate affinity of the enzyme (Ardissone et al. 2005; El Omari et al. 2006; Lee and Goodey 2011). Lee and Goodey have described possible reasons for these indirect effects based on inter molecular relationships, charge distribution and conformational motion of proteins (Lee and Goodey 2011).

While tyrosinase variants exhibited improved activity with D-tyrosine, the engineered enzymes generally exhibited decreases (up to 1.6 fold) in $\mathrm{k}_{\text {cat }}$ with L-tyrosine compared to the WT (Table III). The maximum improvement in catalytic efficiency of the selected variants was 2.2 fold improved over the WT compared to a 16 fold improvement observed for D-tyrosine. However, enzyme variants still exhibited lower $K_{m}$ values with L-tyrosine compared to D-tyrosine. Indeed, the catalytic efficiency of the WT and variant tyrosinases is higher with the L enantiomer compared to the D-enantiomer (Table I and Table III). Thus, the amino acid changes which we observed in these variants have improved the oxidation of D-tyrosine more than L-tyrosine generating a variant (random variant RV145) with a catalytic efficiency $\left(48 \mathrm{~min}^{-1} \mathrm{mM}^{-1}\right)$ higher than the catalytic efficiency of the WT with L-tyrosine (40.4 $\left.\min ^{-1} \mathrm{mM}^{-1}\right)$.

There has been limited characterisation of tyrosinases carried out in the presence of D-tyrosine. When tyrosinase was purified from Agaricus bisporus and immobilised on glycerin cinnamate, the kinetics for D-tyrosine were measured at $46 \mathrm{~s}^{-1}(\mathrm{kcat}), 1.5 \mathrm{mM}\left(K_{m}\right)$, and $30.6 \mathrm{~s}^{-1} \mathrm{mM}^{-1}\left(\mathrm{kcat} / K_{m}\right)$ (Marín-Zamora et al. 2007). This data differed from kinetic studies of free Agaricus bisporus tyrosinase with D-tyrosine which calculated rates at $8 \mathrm{~s}^{-1}$ (kcat), $1.86 \mathrm{mM}\left(K_{m}\right)$ and $4.3 \mathrm{~s}^{-1} \mathrm{mM}^{-1}(\mathrm{kcat} /$ $\left.K_{m}\right)($ Espin et al. 1998; Espin et al. 1997). The catalytic activity of tyrosinase from Neurospora crassa was also measured in the presence of D-tyrosine. The kcat and $\mathrm{km}$ were calculated to be $160 \mathrm{~s}^{-1}$ and $0.32 \mathrm{mM}$ respectively. The $K_{m}$ of $R$. solanacearum variant tyrosinase RV145 for D-tyrosine was 1.5 fold lower than agaricus bisporus tyrosinase but 4 fold higher than tyrosinase from $N$. crassa. The kcat 
values for the best performing tyrosinase variant in the current study (V153A) were 15 fold and 80 fold lower respectively.

While tyrosinase from Bacillus megaterium was subjected to random mutagenesis and a single mutation variant $(\mathrm{R} 209 \mathrm{H})$ was generated with a 1.6 fold increase in monophenolase:diphenolase ratio (MDR) compared to the WT with L-tyrosine and L-DOPA (Ben-Yosef et al. 2010), the catalytic efficiency was reduced 19.8 fold for L-tyrosine. The activity for D-tyrosine by the B. megaterium tyrosinase mutant was not reported (Ben-Yosef et al. 2010), but the relative activity of the WT for Dtyrosine was $20.3 \%$ of the activity for L-DOPA (Shuster and Fishman 2009). By direct comparison, WT tyrosinase from R. solanacearum exhibits a two fold higher activity with D-tyrosine compared to L-DOPA and tyrosinase variants exhibited between 4 and 7 fold higher activity for D-tyrosine compared to L-DOPA. As far as we are aware, $R$. solanacearum tyrosinase is the only tyrosinase reported with a MDR greater than one (Hernandez-Romero et al. 2006). The newly evolved random tyrosinase variant RV145 has a better monophenolase activity and a higher MDR (90:1) compared to the WT enzyme (Table IV).

In conclusion, this study has generated variants of tyrosinase from $R$. solanacearum with up to 16 fold improved catalytic efficiency towards D-tyrosine and a 3 fold increase in MDR. Amino acid changes T183I and N322S affect the affinity of tyrosinase towards D-tyrosine while amino acid change V153A positively affects the turnover number but negatively impacts on the affinity of the enzyme for D-tyrosine. Both antagonistic $\left(\mathrm{k}_{\mathrm{cat}}\right)$ and synergistic $\left(K_{m}\right)$ effects of amino acid changes were observed in random variant RV145. The catalytic activity of the random variants and the best single mutation variant C10_N322S were also improved towards L-tyrosine but the amino acid changes have a greater affect on D-tyrosine oxidation by tyrosinase. The increased rate of reaction combined with the increased monophenolase to diphenolase ratio suggests that tyrosinase variants of $R$. solanacearum are good targets for development as biocatalysts for the production of D-DOPA. 


\section{Acknowledgements}

This project was funded by University College Dublin research demonstratorships. HD was granted by the German Research Foundation (Graduate School 1043). We also thank Even Solem for his help with Chimera.

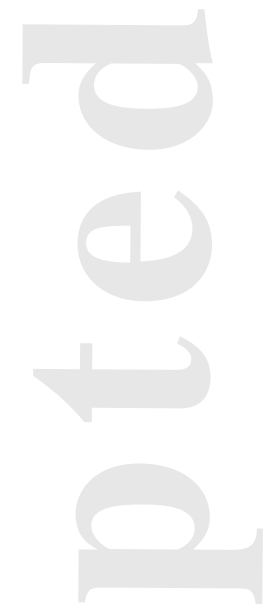




\section{References}

Altschul SF, Madden TL, Schaffer AA, Zhang JH, Zhang Z, Miller W, Lipman DJ. 1997. Gapped BLAST and PSI-BLAST: a new generation of protein database search programs. Nucleic Acids Res 25:3389-3402.

Ardissone S, Laurenti E, Frendo P, Ghibaudi E, Puppo A. 2005. Single-site mutations on the catalaseperoxidase from Sinorhizobium meliloti: role of the distal Gly and the three amino acids of the putative intrinsic cofactor. J Biol Inorg Chem 10:813-826.

Ben-Yosef VS, Sendovski M, Fishman A. 2010. Directed evolution of tyrosinase for enhanced monophenolase/diphenolase activity ratio. Enzyme Micro Technol 47:372-376.

Bennett-Lovsey RM, Hebert AD, Sternberg MJE, Kelley LA. 2008. Exploring the extremes of sequence/structure space with ensemble fold recognition in the program Phyre. Proteins: structure, function, bioinformatics 70:611-625.

Betts MJ, Russell RB. 2003. Amino acid properties and consequences of substitutions. In: Barnes M, Gray IC, editors. Bioinformatics for Geneticists: John Wiley \& Sons, Ltd. p 300-302.

Bloom JD, Arnold FH. 2009. In the light of directed evolution: Pathways of adaptive protein evolution. Proc Natl Acad Sci U S A 106:9995-10000.

Bugg T. 1997. An introduction to enzyme and coenzyme chemistry: Blackwell Publishers Ltd. Oxford, UK. 247 p.

Burton SG. 2003. Oxidizing enzymes as biocatalysts. Trends Biotechnol 21:543-549.

Claus H, Decker H. 2006. Bacterial tyrosinases. Syst Appl Microbiol 29:3-14.

Decker H, Schweikardt T, Tuczek F. 2006. The first crystal structure of tyrosinase: All questions answered? Angew Chem Int Ed 45:4546-4550. 
Decker H, Tuczek F. 2000. Tyrosinase/catecholoxidase activity of hemocyanins: structural basis and molecular mechanism. Trends Biochem Sci 25:392-397.

El Omari K, Liekens S, Bird LE, Balzarini J, Stammers DK. 2006. Mutations distal to the substrate site can affect varicella zoster virus thymidine kinase activity: Implications for drug design. Mol Pharmacol 69:1891-1896.

Engelman DM, Steitz TA, Goldman A. 1986. Identifying nonpolar transbilayer helices in mino-acidsequences of membrane-proteins. Ann Rev Biophys Biophysical Chemi 15:321-353.

Espin J, Soler-Rivas C, Cantos E, Tomas-Barberan FA, Wichers HJ. 2001. Synthesis of the antioxidant hydroxytyrosol using tyrosinase as biocatalyst. J Agricult Food Chem 49:1187-1193.

Espin J, Trujano MF, Tudela J, Garcia-Canovas F. 1997. Monophenolase activity of polyphenol oxidase from Haas avocado. J Agricult Food Chem 45:1091-1096.

Espin JC, Morales M, Garcia-Ruiz PA, Tudela J, Garcia-Canovas F. 1997. Improvement of a continuous spectrophotometric method for determining the monophenolase and diphenolase activities of mushroom polyphenol oxidase. J Agricult Food Chem 45:1084-1090.

Espin JC, Garcia-Ruiz PA, Tudela J, Garcia-Canovas F. 1998a. Study of stereospecificity in mushroom tyrosinase. Biochem J 331:547-551.

Espin JC, Morales M, Garcia-Ruiz PA, Tudela J, Garcia-Canovas F. 1998b. Study of the stereospecificity in pear and strawberry polyphenol oxidases. J Agricult Food Chem 46:24692473.

Garcia-Molina Mdel M, Muñoz-Muñoz JL, Garcia-Molina F, García-Ruiz P, Garcia-Canovas F. 2012. Action of tyrosinase on ortho-substituted phenols: possible influence on browning and melanogenesis. J Agricult Food Chem 60:6447-6453. 
Halaouli S, Asther M, Sigoillot JC, Hamdi M, Lomascolo A. 2006. Fungal tyrosinases: new prospects in molecular characteristics, bioengineering and biotechnological applications. J Appl Microbiol 100:219-232.

Hernandez-Romero D, Sanchez-Amat A, Solano F. 2006. A tyrosinase with an abnormally high tyrosine hydroxylase/dopa oxidase ratio - role of the seventh histidine and accessibility to the active site. FEBS J 273:257-270.

Hernandez-Romero D, Solano F, Sanchez-Amat A. 2005. Polyphenol oxidase activity expression in Ralstonia solanacearum. Appl Environ Microbiol 71:6808-6815.

Hume AR, Nikodinovic-Runic J, O'Connor KE. 2009. FadD from Pseudomonas putida CA-3 is a true long-chain fatty acyl Coenzyme A synthetase that activates phenylalkanoic and alkanoic acids. J Bacteriol 191:7554-7565.

Jus S, Stachel I, Fairhead M, Meyer M, Thöny-Meyer L, Guebitz GM. 2012. Enzymatic cross-linking of gelatine with laccase and tyrosinase. Biocatal Biotransformation 30:86-95

Kawazoe T, Tsuge H, Imagawa T, Aki K, Kuramitsu S, Fukui K. 2007. Structural basis of D-DOPA oxidation by D-amino acid oxidase: Alternative pathway for dopamine biosynthesis. Biochem Biophys Res Commun 355:385-391.

Krieger E, Darden T, Nabuurs S, Finkelstein A, Vriend G. 2004. Making optimal use of empirical energy functions: Force-field parameterization in crystal space. Proteins 57:678-683.

Laemmli UK. 1970. Cleavage of structural proteins during the assembly of the head of bacteriophage T4. Nature 227:680-685.

Larkin MA, Blackshields G, Brown NP, Chenna R, McGettigan PA, McWilliam H, Valentin F, Wallace IM, Wilm A, Lopez R and others. 2007. Clustal W and Clustal X version 2.0. Bioinformatics 23:2947-2948. 
Lee J, Goodey NM. 2011. Catalytic contributions from remote regions of enzyme structure. Chem Rev 111:7595-7624.

Lipski J, Nistico R, Berretta N, Guatteo E, Bernardi G, Mercuri NB. 2011. L-DOPA: A scapegoat for accelerated neurodegeneration in Parkinson's disease? Prog Neurobiol 94:389-407.

Marin-Zamora ME, Rojas-Melgarejo F, García-Cánovas F, García-Ruiz PA. 2007. Stereospecificity of mushroom tyrosinase immobilized on a ciral and a nonchiral support. J Agricult Food Chem $55: 4569-4575$.

Martin LB, Nikodinovic J, Mc Mahon AM, Vijgenboom E, O'Connor KE. 2008. Assessing the catalytic activity of three different sources of tyrosinase: A study of the oxidation of mono- and difluorinated monophenols. Enzyme Microbial Technol 43:297-301.

Matoba Y, Kumagai T, Yamamoto A, Yoshitsu H, Sugiyama M. 2006. Crystallographic evidence that the dinuclear copper center of tyrosinase is flexible during catalysis. J Biol Chem 281:89818990.

Melo F, Šali A. 2007. Fold assessment for comparative protein structure modeling. Protein Sci $16: 2412-2426$.

Mildvan AS, Weber DJ, Kuliopulos A. 1992. Qunatitative interpretations of double mutations of enzymes. Arch Biochem Biophys 294:327-340.

Moses J, Siddiqui A, Silverman PB. 1996. Sodium benzoate differentially blocks circling induced by D- and L-dopa in the hemi-Parkinsonian rat. Neurosci Lett 218:145-148.

Notredame C, Higgins DG, Heringa J. 2000. T-Coffee: A novel method for fast and accurate multiple sequence alignment. J Mol Biol 302:205-217.

Patthy L. 1999. Protein Evolution: Blackwell Publishing Ltd. Oxford, UK. p 12-17.

Petsko GA, Ringe D. 2004. Protein structure and function: New Science Press Ltd. 195 p. 
Rolff M, Schottenheim J, Decker H, Tuczek F. 2011. Copper- $\mathrm{O}_{2}$ reactivity of tyrosinase models towards external monophenolic substrates: molecular mechanism and comparison with the enzyme. Chem Soc Rev 40:4077-4098.

Salanoubat M, Genin S, Artiguenave F, Gouzy J, Mangenot S, Arlat M, Billault A, Brottier P, Camus JC, Cattolico L and others. 2002. Genome sequence of the plant pathogen Ralstonia solanacearum. Nature 415:497-502.

Šali A, Blundell TL. 1993. Comparative protein modelling by satisfaction of spatial restraints. J Mol Biol 234:779-815.

Salverda MLM, Dellus E, Gorter FA, Debets AJM, van der Oost J, Hoekstra RF, Tawfik DS, de Visser JAGM. 2011. Initial mutations direct alternative pathways of protein evolution. PLoS Genet 7:e1001321.

Sambrook J, Fritsch EF, Maniatis T. 1989. Molecular cloning: A laboratory manual. Cold Spring Harbor Laboratory: Cold Spring Harbor, NY.

Sánchez-Ferrer A, Rodriguezlopez J, Garciacanovas F, Garciacarmona F. 1995. Tyrosinase - a comprehensive review of its mechanism. Biochim Biophys Acta-Protein Struct Molec Enzymol $1247: 1-11$

Shuster V, Fishman A. 2009. Isolation, cloning and characterization of a tyrosinase with improved activity in organic solvents from Bacillus megaterium. J Molec Microbiol Biotechnol 17:188200.

Smith PK, Krohn RI, Hermanson GT, Mallia AK, Gartner FH, Provenzano MD, Fujimoto EK, Goeke NM, Olson BJ, Klenk DC. 1985. Measurement of protein using bicinchoninic acid. Analyt Biochem 150:76-85.

Solomon EI, Sundaram UM, Machonkin TE. 1996. Multicopper oxidases and oxygenases. Chem Rev $96: 2563-2605$. 
van Holde KE, Miller KI, Decker H. 2001. Hemocyanins and invertebrate evolution. J Biol Chem 276:15563-15566.

Wilcox DE, Porras AG, Hwang YT, Lerch K, Winkler ME, Solomon EI. 1985. Substrate-analog binding to the coupled binuclear copper active-site in tyrosinase. J Amer Chem Soc 107:40154027.

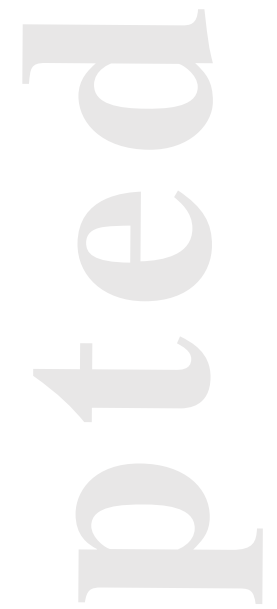




\section{Figure Legends}

Figure 1. The sequence of ortho hydroxylation of phenolic compounds by monophenolase activity and subsequent oxidation of the $o$-diphenolic product by diphenolase activity of tyrosinase.

Figure 2. Schematic overview of single mutations introduced into WT based on RVC10 variant amino acid changes (A); Schematic overview of single mutations introduced into WT based on RV145 variant amino acid changes (B).

Figure 3. A) Three different models of the WT tyrosinase. Model 1 (yellow) is based on an alignment with Clustal, model 2 (blue) on an alignment with Align2d and model 3 (green) on an alignment with T-coffee. The active site with two copper atoms (magenta) and the six coordinating histidines are shown only for model 1 . Amino acids mutated in this study are drawn with balls and sticks and are labelled. For visual clarity the trace for amino acid ranges 155-173 and 237-245 are not shown. B) and C) Close-up view of the region near the mutation site V153A for model 1. P247 is located at the Nterminal end of a helix which contains $\mathrm{H} 253$, one of the coordinating histidines of $\mathrm{CuB}$. Carbon atoms are drawn in yellow, nitrogen in blue and oxygen atoms in red. Images were generated with YASARA (Krieger et al. 2004). 
Table I. Kinetic constants characterising reactions of purified tyrosinases with D-tyrosine as substrate using wild type (WT) and random mutation enzyme variants RVC10 and RV145.

\begin{tabular}{c|ccc}
\hline Variant & $\mathbf{k}_{\text {cat }}\left(\mathbf{m i n}^{-\mathbf{1}}\right)$ & $\boldsymbol{K}_{\boldsymbol{m}}(\mathbf{m M})$ & $\begin{array}{c}\mathbf{k}_{\text {cat }} / \boldsymbol{K}_{\boldsymbol{m}} \\
\left(\mathbf{m i n}^{-\mathbf{1}} \mathbf{m M}^{-\mathbf{1}}\right)\end{array}$ \\
\hline WT & $18 \pm 0.6$ & $6.2 \pm 0.1$ & 3 \\
RVC10 & $38 \pm 1.2$ & $1.9 \pm 0.1$ & 20 \\
RV145 & $58 \pm 2.2$ & $1.2 \pm 0.3$ & 48 \\
\hline
\end{tabular}


Table II. Kinetic constants of purified single mutation variants of tyrosinase compared to the WT and random variants RVC10 and RV145 with D-tyrosine as the substrate ${ }^{\mathrm{a}}$. Single amino acid changes were introduced into the WT by SDM.

\begin{tabular}{cccc}
\hline Variant & $\begin{array}{c}\mathbf{k}_{\mathbf{c a t}} \\
\left(\mathbf{m i n}^{-\mathbf{1}}\right)\end{array}$ & $\begin{array}{c}\boldsymbol{K}_{\boldsymbol{m}} \\
\mathbf{( m M )}\end{array}$ & $\begin{array}{c}\mathbf{k}_{\mathbf{c a t}} / \boldsymbol{K}_{\boldsymbol{m}} \\
\left(\mathbf{m i n}^{-\mathbf{1}} \mathbf{m} \mathbf{M}^{-\mathbf{1}} \mathbf{)}\right.\end{array}$ \\
\hline WT & $18 \pm 0.6$ & $6.2 \pm 0.1$ & 2.9 \\
RVC10 & $38 \pm 1.2$ & $1.9 \pm 0.1$ & 20.0 \\
RV145 & $58 \pm 2.2$ & $1.2 \pm 0.3$ & 48.3 \\
C10_T183I & $82 \pm 3$ & $3.5 \pm 0.3$ & 23.4 \\
C10_F185Y & $102 \pm 6$ & $7.3 \pm 0.8$ & 13.9 \\
C10_N322S & $73 \pm 6$ & $2.4 \pm 0.5$ & 30.4 \\
C10_T359M & $82 \pm 5$ & $6.3 \pm 0.9$ & 13.0 \\
145_Y119F & $76 \pm 4$ & $5.1 \pm 0.5$ & 14.9 \\
145_V153A & $126 \pm 4.7$ & $15.0 \pm 0.9$ & 8.4 \\
145_D317Y & $81 \pm 4.6$ & $6.9 \pm 0.6$ & 11.8 \\
145_L330V & $98 \pm 4.8$ & $9.6 \pm 0.3$ & 10.3 \\
\hline
\end{tabular}

${ }^{a}$ Data is an average of three independent determinations. 
Table III. Kinetic constants characterising the reactions of purified tyrosinase from the WT, random mutation variants and the best D-tyrosine single mutation variant using L-tyrosine as the substrate ${ }^{\mathrm{a}}$. Data for WT and tyrosinase variants with D-tyrosine is shown in the grey column.

\begin{tabular}{|c|c|c|c|c|}
\hline \multirow[b]{2}{*}{ Variant } & \multicolumn{3}{|c|}{ L-tyrosine } & D-tyrosine \\
\hline & $k_{\text {cat }}\left(\min ^{-1}\right)$ & $K_{m}(\mathbf{m M})$ & $\begin{array}{c}\mathbf{k}_{\mathrm{cat}} / K_{m} \\
\left(\mathbf{m i n}^{-1} \mathbf{m M}^{-1}\right) \\
\end{array}$ & $\begin{array}{c}\mathbf{k}_{\text {cat }} / K_{m} \\
\left(\mathrm{~min}^{-1} \mathbf{m M}^{-1}\right) \\
\end{array}$ \\
\hline WT & $85 \pm 6$ & $2.1 \pm 0.4$ & 40.4 & 2.9 \\
\hline RVC10 & $62 \pm 8$ & $0.9 \pm 0.3$ & 67 & 20.0 \\
\hline RV145 & $55 \pm 3.5$ & $0.6 \pm 0.09$ & 85.2 & 48.3 \\
\hline C10_N322S & $74.2 \pm 5$ & $1.0 \pm 0.19$ & 74.2 & 30.4 \\
\hline
\end{tabular}

${ }^{\mathrm{a}}$ Data is an average of three independent determinations. 
Table IV. The ratio of monophenolase to diphenolase activity of the WT tyrosinase, random mutation variants and the single mutation variant with the highest catalytic efficiency with D-tyrosine ${ }^{a}$.

\begin{tabular}{c|ccc|cc}
\hline \multicolumn{1}{c}{ D-tyrosine : D-DOPA } & \multicolumn{2}{c}{ L-tyrosine : L-DOPA } \\
\hline Strain & $\begin{array}{c}\text { Monophenolase } \\
\text { (nmoles/min/mg protein) }\end{array}$ & $\begin{array}{c}\text { Diphenolase } \\
\text { (nmoles/min/mg protein) }\end{array}$ & Ratio & $\begin{array}{c}\text { Monophenolase } \\
\text { (nmoles/min/mg protein) }\end{array}$ & $\begin{array}{c}\text { Diphenolase } \\
\text { (nmoles/min/mg protein) }\end{array}$ \\
\hline Ratio \\
WT & $1650 \pm 121$ & $54.4 \pm 5$ & $30: 1$ & $9720 \pm 460$ & $840 \pm 123$ \\
RVC10 & $5000 \pm 240$ & $82 \pm 1$ & $61: 1$ & $12840 \pm 820$ & $820 \pm 162$ \\
RV145 & $9133 \pm 460$ & $102 \pm 7$ & $90: 1$ & $20900 \pm 2651$ & $1240 \pm 211$ \\
C10_N322S & $4100 \pm 577$ & $270 \pm 13$ & $15: 1$ & $16400 \pm 1637$ & $17: 1$ \\
\hline
\end{tabular}

${ }^{a}$ Data is an average of three independent determinations. 


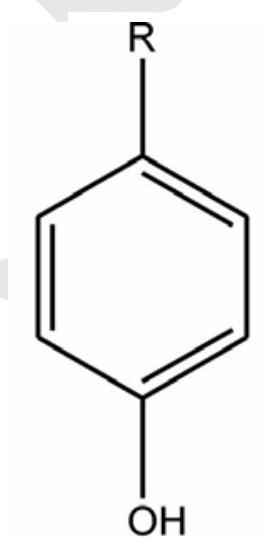

Monophenol

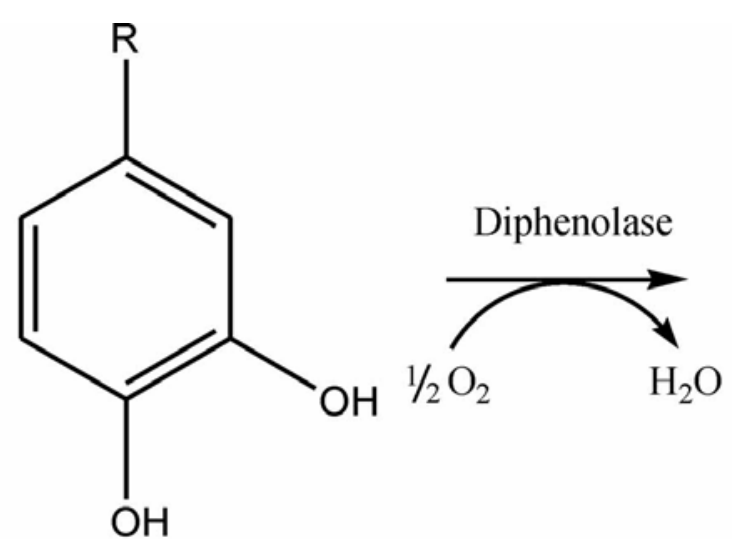

$o$-Diphenol

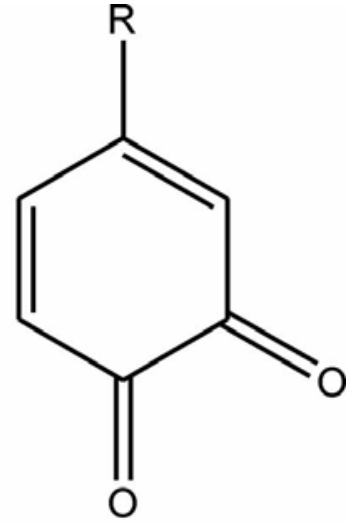

$o$-Quinone

Figure 1 

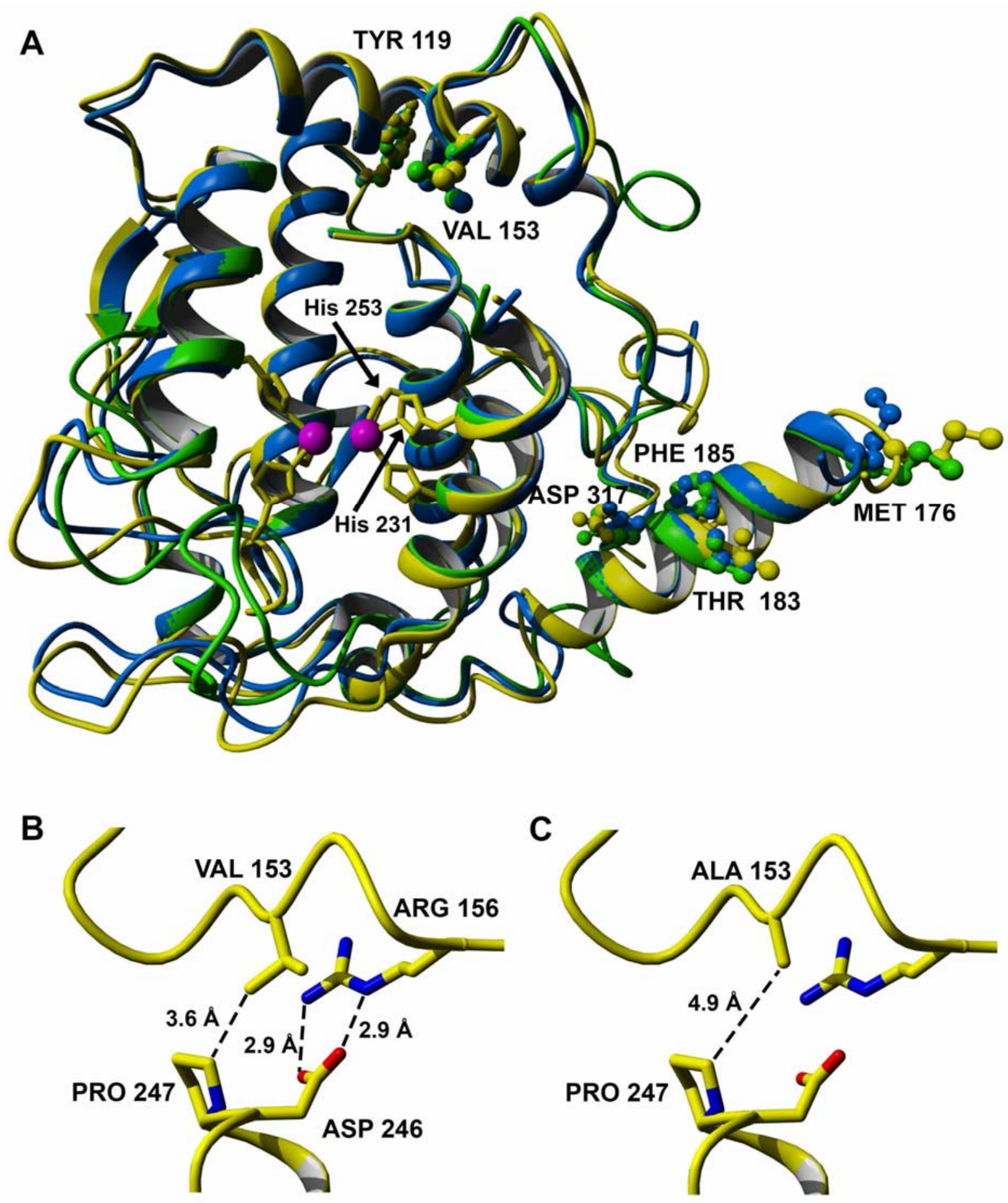

Figure 3 